\title{
Eriodictyol attenuates spinal cord injury by activating Nrf2/HO-1 pathway and inhibiting NF-kB pathway
}

\author{
Xiaojie Mao', Zhiyang Jiang ${ }^{1 *}$, Chaohong Shi ${ }^{1}$, Junjun Lu², Gaofeng Rao ${ }^{1}$ \\ ${ }^{1}$ Department of Rehabilitation Medicine, '2Department of Nephrology, The First People's Hospital of Wenling, Taizhou City, \\ Zhejiang Province 317500, China \\ *For correspondence: Email: FDRT98Ilo@163.com; Tel: +86-576-89668302
}

Sent for review: 8 May 2020

Revised accepted: 28 July 2020

\begin{abstract}
Purpose: To investigate the effect of eriodictyol on spinal cord injury (SCI) and its underlying mechanism of action.

Methods: Thirty Sprague-Dawley rats were assigned to sham, SCl, and eriodictyol-treated groups (SCI

+ Eri; 10, 20, and $50 \mathrm{mg} / \mathrm{kg}$ ). Moderate spinal cord contusion injury was induced to model SCl. Locomotor recovery was assessed based on Basso, Beattie, and Bresnahan (BBB) score. Pain was evaluated by paw withdrawal threshold (PWT) and latency (PWL), and spinal cord water content was measured. Tumor necrosis factor-alpha (TNF- $\alpha$ ), interleukin-1 beta (IL-1 $\beta$ ), and interleukin-6 (IL-6) expression were determined by enzyme-linked immunosorbent assay (ELISA) and reverse transcriptase quantitative polymerase chain reaction (RT-qPCR). Immunoassay was used to determine malondialdehyde (MDA), superoxide dismutase (SOD), glutathione (GSH), and glutathione peroxidase (GSH-PX) levels while Western blotting was employed to evaluate nuclear factor erythroid 2-related factor 2 (Nrf2), heme oxygenase-1 (HO-1), nuclear factor-kappa B (NF-kB), and phosphorylated NF-kB (p-NF-KB) levels.

Results: Eriodictyol elevated BBB score, PWT, and PWL in SCl rats but reduced spinal cord water content $(p<0.05)$. Eriodictyol treatment down-regulated TNF- $\alpha, I L-1 \beta, I L-6$, and MDA, whereas SOD, $\mathrm{GSH}$, and GSH-PX levels were elevated $(p<0.05)$. Eriodictyol administration increased Nrf2 and HO-1 levels but reduced $p-N F-K B / N F-K B$.

Conclusion: This study provides a potential therapy to promote long-term functional recovery following $\mathrm{SCl}$.
\end{abstract}

Keywords: Spinal cord injury, Eriodictyol, Nrf2/HO-1 pathway, NF-kB signaling pathway, Polymerase chain reaction, Basso, Beattie and Bresnahan score

\begin{abstract}
This is an Open Access article that uses a fund-ing model which does not charge readers or their institutions for access and distributed under the terms of the Creative Commons Attribution License (http://creativecommons.org/licenses/by/4.0) and the Budapest Open Access Initiative (http://www.budapestopenaccessinitiative.org/read), which permit unrestricted use, distribution, and reproduction in any medium, provided the original work is properly credited.

Tropical Journal of Pharmaceutical Research is indexed by Science Citation Index (SciSearch), Scopus, International Pharmaceutical Abstract, Chemical Abstracts, Embase, Index Copernicus, EBSCO, African Index Medicus, JournalSeek, Journal Citation Reports/Science Edition, Directory of Open Access Journals (DOAJ), African Journal Online, Bioline International, Open-J-Gate and Pharmacy Abstracts
\end{abstract}

\section{INTRODUCTION}

Car accidents and falls frequently cause spinal cord injury (SCl), a devastating and common condition of acute trauma [1]. This trauma can impair bowel and bladder function, mobility, and autonomic nerve function and is often accompanied by pressure ulcers and pain that directly damages patient health [2]. In terms of pathophysiology, secondary injuries that may occur after $\mathrm{SCl}$ include edema, secondary ischemia, oxidative stress injury, inflammatory 
cell infiltration, and neuronal apoptosis [3-5]. Though spinal surgery and methylprednisolone are key interventions for SCl [4], there are currently no effective strategies for neurologic or functional recovery following $\mathrm{SCl}$. Therefore, it is critical to develop new therapeutic strategies to promote functional recovery in patients with $\mathrm{SCl}$.

Nuclear factor-kappa B (NF-kB), heme oxygenase-1 (HO-1), nuclear factor erythroid 2related factor 2 (Nrf2), tumor necrosis factoralpha (TNF- $\alpha$ ), interleukin-1 beta (IL-1 $\beta$ ), and interleukin-6 (IL-6) play pivotal roles in oxidative stress and neuro-inflammation [6]. Though NF$\mathrm{KB}$ and $\mathrm{HO}-1 / \mathrm{Nrf} 2$ signaling have been shown to contribute to inflammatory and antioxidant responses after lipopolysaccharide (LPS) challenge [7], the roles of NF-KB and $\mathrm{HO}-1 / \mathrm{Nrf2}$ in $\mathrm{SCl}$ have not been clearly elucidated.

Eriodictyol, a compound isolated from the plant Dracocephalum rupestre, is widely distributed in common foods [8]. Published studies have demonstrated that eriodictyol has a variety of biological effects, including the suppression of oxidative stress, inflammation, cell apoptosis, and osteoclast-related diseases [9]. Eriodictyol has been shown to elevate $\mathrm{HO}-1$ levels by activating the Nrf2/antioxidant response element (ARE) pathway and to protect against hydrogen peroxide-induced neurotoxicity [10]. In LPSinduced neuro-inflammation, eriodictyol blocked downstream translocation of NF-KB and thus attenuated amyloidogenesis and memory impairment [11]. However, the effect of eriodictyol on $\mathrm{SCl}$ and its molecular mechanism are still poorly understood.

The present study demonstrates the protective properties of eriodictyol against $\mathrm{SCl}$ in rats and investigates the mechanism underlying its protective effects. This study identifies eriodictyol as a potential therapeutic strategy for patients with SCl.

\section{EXPERIMENTAL}

\section{Animals}

A total of 30 male Sprague-Dawley rats (8 - 10 weeks old; $250 \pm 20 \mathrm{~g}$ ) were bought from the animal breeding center of Fujian Medical University. The rats were maintained at $25 \pm 2{ }^{\circ} \mathrm{C}$ for 3 days with food and water ad libitum before experiments. All experimental procedures conformed to the guidelines of National Institutes of Health Guide for The Care and Use of Laboratory Animals [12], and the study was approved by the Ethics Committee of The First
People's Hospital of Wenling (approval no. 20170046).

\section{Establishment of SCI model and treatment}

Rats were randomly assigned to sham operation (sham), SCl, and eriodictyol-treated (SCl + Eri, 10,20 , and $50 \mathrm{mg} / \mathrm{kg}$ ) groups. The $\mathrm{SCl}$ model was established using a spinal cord contusion injury [13]. Animals were anesthetized by intraperitoneal administration of $50 \mathrm{mg} / \mathrm{kg}$ sodium pentobarbital (Sigma, St. Louis, MO, USA). Moderate contusion injury was induced with a laminectomy of the eighth thoracic vertebra (T8). The T8 spinous process and laminae were excised to expose circular dura with a diameter of $2.4 \mathrm{~mm}$, and a $2 \mathrm{~g}$ weight was dropped from a height of $5 \mathrm{~cm}$ onto the exposed dura. After injury, the overlying muscles and skin were sewn closed. Laminectomy without compression was performed in the sham operation group.

Rats were administered 10,20 , or $50 \mathrm{mg} / \mathrm{kg}$ of eriodictyol (Seebio Biotech Co. Ltd., Shanghai, China) daily for 4 weeks. The sham group was treated with saline. On day 28 , the rats were anesthetized and sacrificed.

\section{Evaluation of locomotor function recovery and behavioral pain tests}

Basso, Beattie, and Bresnahan (BBB) scores were assigned as a measure of locomotor recovery. The locomotor rating scale was $0-21$, where a score of " 0 " indicated no visible hind limb movement and a score of "21" indicated normal movement.

Paw withdrawal threshold (PWT) was measured to assess mechanical allodynia. Paw withdrawal latency (PWL) was determined using the Hargreaves method of responding to radiant heat. The PWT and PWL assessments were performed as previously reported [14].

\section{Assessment of spinal cord water content}

At $72 \mathrm{~h}$ post-injury, the spinal cord was obtained from the epicenter for water content assessment. The wet weight of the spinal cord sample was measured, and dry weight was measured after $48 \mathrm{~h}$ of drying at $80^{\circ} \mathrm{C}$. Percentage of spinal cord water content was calculated by the following equation: (wet weight - dry weight) / wet weight $\times$ $100 \%$.

\section{Determination of cytokine expression}

Peripheral blood was obtained after eriodictyol treatment and subjected to centrifugation at 
$10,000 \times \mathrm{g}$ for $10 \mathrm{~min}$ at $4^{\circ} \mathrm{C}$ to obtain cell-free supernatants. The levels of TNF- $\alpha, \mathrm{IL}-1 \beta$, and IL6 were determined using ELISA kits (Dakewe Biotech, Shenzhen, China) according to the manufacturer's instructions.

Reverse $\quad$ transcriptase $\quad$ quantitative
polymerase chain reaction (RT-qPCR)

Total RNA was extracted using Trizol reagent (Gibco BRL, Grand Island, NY, USA). PCR was conducted on an ABI 7500 Real-Time PCR System. The RT-qPCR primer pairs are shown in Table 1. The PCR was performed as follows: 95 ${ }^{\circ} \mathrm{C}$ for $10 \mathrm{~min}, 90{ }^{\circ} \mathrm{C}$ for $15 \mathrm{~s}$ and $60{ }^{\circ} \mathrm{C}$ for $60 \mathrm{~s}$ for 40 cycles, $95^{\circ} \mathrm{C}$ for $60 \mathrm{~s}$.

Determination of malondialdehyde (MDA), superoxide dismutase (SOD), glutathione (GSH), and glutathione peroxidase (GSH-PX) levels

Peripheral blood was obtained after eriodictyol treatment and subjected to centrifugation at $10,000 \times \mathrm{g}$ for $10 \mathrm{~min}$ at $4{ }^{\circ} \mathrm{C}$. The levels of MDA, SOD, GSH, and GSH-PX were determined using immunoassay kits (Dakewe Biotech).

\section{Western blot analysis}

Spinal cord samples (15 mg) were homogenized, and proteins were extracted on ice with lysis buffer (Thermo Scientific, Rockford, IL, USA). Cells were washed twice with cold PBS. After sonication and centrifugation, protein concentration in the supernatant was determined using the Bicinchoninic Acid Protein Assay Kit (Thermo). Equal amounts of total protein were separated by $10 \%$ SDS-PAGE, and the separated proteins were transferred onto polyvinylidene difluoride membranes. Nonspecific binding sites were blocked in $\mathrm{NaCl} / \mathrm{T}$ ris- $\mathrm{T}$ buffer containing $5 \%$ non-fat milk for $1 \mathrm{~h}$.

Membranes were incubated overnight at $4{ }^{\circ} \mathrm{C}$ with primary antibodies, including monoclonal mouse anti-human antibodies against Nrf2, HO1, NF-kB, and phosphorylated NF-kB (p-NF-kB) (BD Biosciences Franklin Lakes, NJ, USA). The membranes were then incubated for $1 \mathrm{~h}$ at room temperature with anti-mouse IgG conjugated with horseradish peroxidase (BD Biosciences). Protein expression levels were determined using an enhanced chemiluminescence detection system (GE Healthcare, USA).

\section{Statistical analysis}

SPSS 17.0 (SPSS Inc, Chicago, IL, USA) was used for all statistical analyses. The experimental data were expressed as means \pm SD. Comparisons among multiple groups were performed using ANOVA followed by Tukey's multiple comparison test. The cutoff for statistical significance was $p<0.05$.

\section{RESULTS}

\section{Eriodictyol alleviated locomotor dysfunction in $\mathrm{SCl}$ rats}

Different concentrations of eriodictyol were administered after $\mathrm{SCl}$ induction in rats. Locomotor function recovery was evaluated using BBB scores. The $\mathrm{SCl}$ group exhibited significantly lower BBB scores than the sham group ( $p<0.01$ ), whereas eriodictyol treatment significantly elevated BBB scores compared to the untreated $\mathrm{SCl}$ group $(p<0.01)$ (Figure $1 \mathrm{~A}$ ). In addition, $\mathrm{SCl}$ induction led to a decrease in PWT and PWL compared to the sham group ( $p<$ $0.01)$, whereas eriodictyol treatment increased both measures relative to the untreated $\mathrm{SCl}$ group $(p<0.01)$ (Figure $1 \mathrm{~B}$ and $\mathrm{C}$ ). The spinal cord water content of $\mathrm{SCl}$ rats was increased compared to the sham group $(p<0.01)$, whereas eriodictyol administration significantly decreased spinal cord water content compared to the untreated $\mathrm{SCl}$ group $(p<0.01)$ (Figure $1 \mathrm{D})$.

\section{Eriodictyol attenuates pro-inflammation cytokines in $\mathrm{SCl}$ rats}

Levels of TNF- $\alpha$, IL-1 $1 \beta$, and IL- 6 were significantly up-regulated in $\mathrm{SCl}$ rats compared to the sham group $(p<0.01)$. However, eriodictyol administration significantly inhibited proinflammatory cytokine up-regulation compared to the untreated SCI group $(p<0.01)$ (Figure 2).

Table 1: Primer pairs used for RT-qPCR

\begin{tabular}{cll}
\hline & & \multicolumn{1}{c}{ Primer sequence } \\
\hline IL-6 & forward & 5'-CCA GAA ACC GCT ATG AAG TTCC-3' \\
& reverse & 5'-TCA CCA GCA TCA GTC CCA AG-3' \\
TNF- $\alpha$ & forward & 5'-CTC CAG GCG GTG CCT ATGT-3' \\
& reverse & 5'-GAA GAG CGT GGT GGC CC-3' \\
IL-1 $\beta$ & forward & 5'-CAA CCA ACA AGT GAT ATT CTC CATG-3' \\
& reverse & 5'-GAT CCA CAC TCT CCA GCT GCA-3' \\
\hline
\end{tabular}




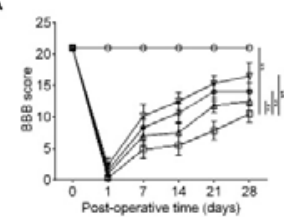

C

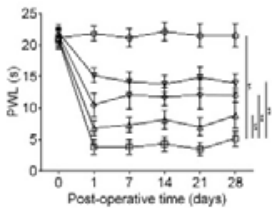

D

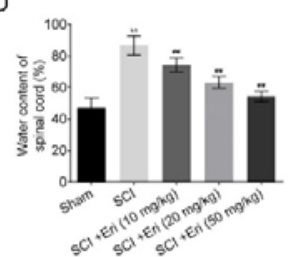

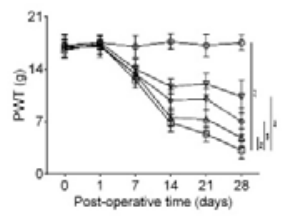

Figure 1: Eriodictyol alleviates motor dysfunction in $\mathrm{SCl}$ rats. (A) BBB scores in sham, $\mathrm{SCl}$, and eriodictyol-treated groups; (B) PWT assessments in sham, $\mathrm{SCl}$, and eriodictyol-treated groups; (C) PWL measurements in sham, $\mathrm{SCl}$, and eriodictyol-treated groups; (D), Assessments of spinal cord water contents in sham, $\mathrm{SCl}$, and eriodictyol-treated groups. o, sham;, SCl; $\triangle, \mathrm{SCl}+$ Eri $(10 \mathrm{mg} / \mathrm{kg}) ; \diamond, \mathrm{SCl}+$ Eri $(20 \mathrm{mg} / \mathrm{kg}) ; \nabla, \mathrm{SCl}+\operatorname{Eri}(50 \mathrm{mg} / \mathrm{kg}) ;{ }^{* *} p<0.01$, compared to the sham group; ${ }^{\#} p<0.01$, compared to the $\mathrm{SCl}$ group

A

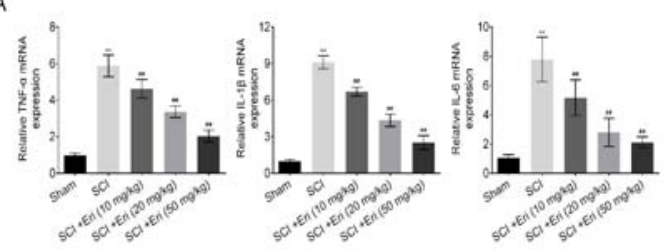

B

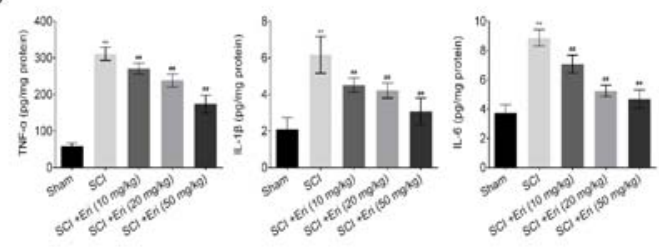

Figure 2: Eriodictyol attenuates pro-inflammatory cytokines in $\mathrm{SCl}$ rats. ${ }^{* *} p<0.01$, compared to the sham group; ${ }^{\#} p<0.01$, compared to the SCl group

\section{Eriodictyol attenuates oxidative stress in $\mathrm{SCl}$ rats}

Induction of $\mathrm{SCl}$ led to increased MDA expression compared to the sham group $(p<$ 0.01 ), whereas eriodictyol treatment significantly decreased MDA expression compared to untreated $\mathrm{SCl}$ rats $(p<0.01)$ (Figure $3 \mathrm{~A})$. In contrast, $\mathrm{SCl}$ rats exhibited a significant decrease in SOD, GSH, and GSH-PX levels compared to the sham group $(p<0.01)$, whereas eriodictyol treatment gradually up-regulated SOD, GSH, and GSH-PX compared to the untreated $\mathrm{SCl}$ group $(p<0.01)$ (Figure $3 \mathrm{~B}-\mathrm{D})$.
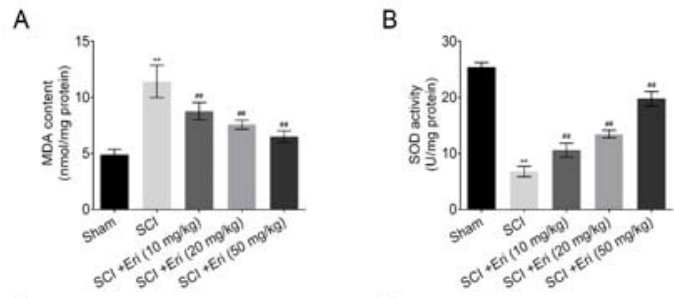

C
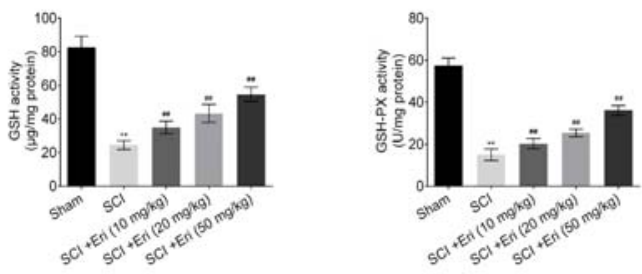

Figure 3: Eriodictyol inhibits oxidative stress in $\mathrm{SCl}$ rats. (A) MDA content in sham, $\mathrm{SCl}$, and eriodictyoltreated groups; (B) SOD activity in sham, $\mathrm{SCl}$, and eriodictyol-treated groups; (C) GSH activity in sham, $\mathrm{SCl}$, and eriodictyol-treated groups; (D) GSH-PX activity in sham, $\mathrm{SCl}$, and eriodictyol-treated groups; ${ }^{* *} p<0.01$, compared to the sham group; ${ }^{\# \#} p<0.01$, compared to the $\mathrm{SCl}$ group

\section{Eriodictyol regulates Nrf2/HO-1 and NF-KB pathways}

Induction of SCl led to elevated $\mathrm{Nrf2}, \mathrm{HO}-1$, and $\mathrm{p}-\mathrm{NF}-\mathrm{kB} / \mathrm{NF}-\mathrm{kB}$ levels compared to the sham group $(p<0.01)$. Eriodictyol treatment (especially 20 and $50 \mathrm{mg} / \mathrm{kg}$ ) significantly increased levels of Nrf2 and HO-1 $(p<0.01)$ but reduced the level of p-NF-KB/NF-KB compared to the untreated SCl group (Figure 4).
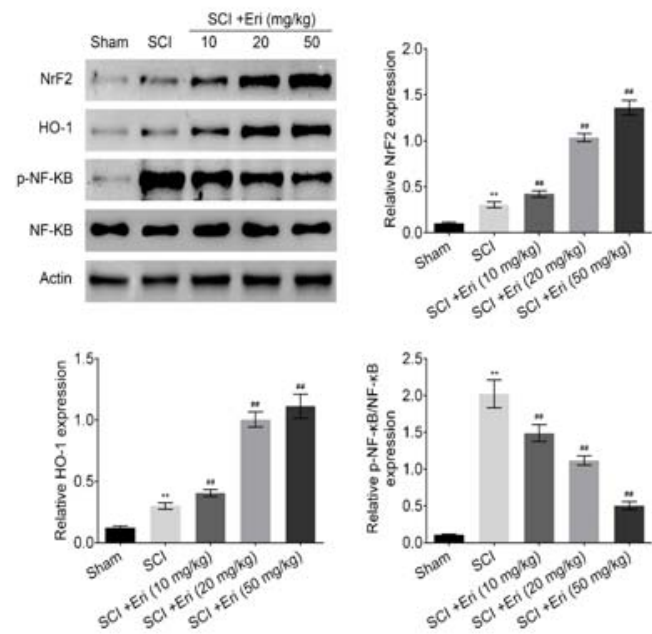

Figure 4: Eriodictyol regulates Nrf2/HO-1 and NF-kB signaling pathways in $\mathrm{SCl}$ rats. ${ }^{* *} P<0.01$, compared to the sham group; ${ }^{\#} p<0.05$ and ${ }^{\# \#} p<0.01$, compared to the $\mathrm{SCl}$ group 


\section{DISCUSSION}

Permanent disability and decreased quality of life and life expectancy can result from SCI [15]. Compelling evidence has shown that functional impairments following $\mathrm{SCl}$ result not only from the initial mechanical damage of the tissue but also from the development of complex secondary events that cause further cell damage [13]. Understanding how complex secondary injuries occur will facilitate the development of effective therapeutic strategy for patients with SCI. Therefore, this study investigated the protective effect of eriodictyol on $\mathrm{SCl}$ and its underlying mechanism. In this study, eriodictyol treatment alleviated locomotor dysfunction and decreased spinal cord water content following $\mathrm{SCl}$. Eriodictyol treatment after $\mathrm{SCl}$ also reduced the expression of pro-inflammatory cytokines, including TNF- $\alpha, I L-1 \beta$, and IL- 6 , and attenuated oxidative stress by decreasing MDA and increasing SOD, GSH, and GSH-PX levels. Moreover, eriodictyol treatment after $\mathrm{SCl}$ increased the expression of Nrf2 and $\mathrm{HO}-1$ and decreased $\mathrm{p}-\mathrm{NF}-\mathrm{kB} / \mathrm{NF}-\mathrm{kB}$ expression, indicating that eriodictyol may alleviate $\mathrm{SCl}$ via regulation of the Nrf2/HO-1 and NF-kB signaling pathways.

Inflammatory responses after trauma are likely to mediate early secondary injuries following $\mathrm{SCI}$ $[13,16]$. It has been reported that levels of IL-1 $\beta$, IL-6, and TNF- $\alpha$ are remarkably elevated following severe $\mathrm{SCl}$, reaching their highest levels at $6 \mathrm{~h}$ post-injury [17]. In an SCl mice model, significant up-regulation of TNF- $\alpha, \mathrm{IL}-1 \beta$, and IL-6 were observed, whereas curcumin administration markedly inhibited the inflammatory response [18]. Oxidative stress and TNF- $\alpha, I L-1 \beta$, and IL-6 levels increased following $\mathrm{SCl}$ [19]. It has been reported that activation of the canonical NF-kB pathway following $\mathrm{SCl}$ indicates activation of the inflammatory response and that transplantation of neural precursors attenuates inflammation by inhibiting the NF-KB pathway [20]. Chlorogenic acid exerts anti-inflammatory effects via inactivating the Tolllike receptor-4/NF-kB and p38 pathways [21]. Consistent with these results, the present study demonstrated that $\mathrm{SCl}$ leads to increased levels of TNF- $\alpha, \quad \mathrm{IL}-1 \beta, \quad \mathrm{IL}-6$, and $p-N F-k B / N F-k B$ relative to the sham group, whereas eriodictyol administration reduces TNF- $\alpha, \mathrm{IL}-1 \beta$, IL- 6 , and $p-$ $N F-\kappa B / N F-k B$ levels relative to the untreated $S C I$ group. These results indicate that eriodictyol inhibits $\mathrm{SCl}$-induced pro-inflammatory cytokines by suppressing the NF-kB pathway.

An earlier study showed that salvianolic acid A alleviates oxidative stress through activation of the Nrf2/HO-1 axis [22]. In a cisplatin-induced nephrotoxicity rat model, epigallocatechin-3gallate increases antioxidant and GSH activates via activating the $\mathrm{Nrf} / \mathrm{HO}-1$ pathway and reduces the inflammatory response by inhibiting NF-kB [23]. In traumatic brain injury mice, protein levels of IL-1 $1 \beta$, IL6, and NF-KB decrease, whereas allyl isothiocyanate administration increases Nrf2 expression, indicating that oxidative stress and inflammation are alleviated via activating the Nrf2/HO-1 pathway or suppressing NF-kB pathway [24].

Tanshinone IIA treatment down-regulates MDA, elevates GSH levels, and attenuates oxidative stress via activating the DJ-1/Nrf2/HO-1 pathway [25]. Moreover, eriodictyol exerts a protective effect on endothelial cells by eliminating oxidative stress-induced cell death via regulation of extracellular signal-regulated kinase (ERK)/Nrf2/ARE-dependent HO-1 expression [26]. The present study found that eriodictyol treatment reduced MDA levels and increased SOD, GSH, and GSH-PX levels. Further, eriodictyol visibly increased $\mathrm{Nrf} 2$ and $\mathrm{HO}-1$ levels, indicating that eriodictyol inhibits oxidative stress following $\mathrm{SCl}$ via activating the Nrf2/HO-1 pathway.

The results of this study show that eriodictyol exerts a protective effect on $\mathrm{SCl}$ in rats via modulation of the NrF2/HO-1 and NF-KB signaling pathways. Further research into the clinical potential of this compound should be conducted. In addition, the mechanism by which eriodictyol attenuates $\mathrm{SCl}$ through regulating Nrf2/HO-1 and NF-kB signaling pathways warrants future investigation.

\section{CONCLUSION}

The findings of this study demonstrate that eriodictyol alleviates $\mathrm{SCl}$ in rats by regulating Nrf2/HO-1 and NF-kB signaling pathways. Thus, the results support the need further investigation of eriodictyol as a potential therapeutic strategy for patients with SCl.

\section{DECLARATIONS}

\section{Conflict of interest}

No conflict of interest is associated with this work.

\section{Contribution of authors}

We declare that this work was done by the authors named in this article and all liabilities 
pertaining to claims relating to the content of this article will be borne by the authors.

\section{Open Access}

This is an Open Access article that uses a funding model which does not charge readers or their institutions for access and distributed under the terms of the Creative Commons Attribution License (http://creativecommons.org/licenses/by/ 4.0) and the Budapest Open Access Initiative (http://www.budapestopenaccessinitiative.org/rea d), which permit unrestricted use, distribution, and reproduction in any medium, provided the original work is properly credited.

\section{REFERENCES}

1. Li J, Liu G, Zheng Y, Hao C, Zhang Y, Wei B, Zhou H, Wang $D$. The epidemiological survey of acute traumatic spinal cord injury (ATSCl) of 2002 in Beijing municipality. Spinal Cord 2011; 49(7): 777-782.

2. Simpson LA, Eng JJ, Hsieh JT, Wolfe, the Spinal Cord Injury Rehabilitation Evidence Research Team DL. The health and life priorities of individuals with spinal cord injury: a systematic review. J Neurotraum 2012; 29(8): 1548-1555.

3. Pei J-p, Fan L-h, Nan K, Li J, Dang X-q, Wang K-z. HSYA alleviates secondary neuronal death through attenuating oxidative stress, inflammatory response, and neural apoptosis in SD rat spinal cord compression injury. J neuroinflamm 2017; 14(1): 97.

4. Ahuja CS, Martin AR, Fehlings M. Recent advances in managing a spinal cord injury secondary to trauma. F1000Research 2016; 5.

5. Sun $X$, Jones $Z B$, Chen $X-m$, Zhou $L$, So $K-F$, Ren $Y$. Multiple organ dysfunction and systemic inflammation after spinal cord injury: a complex relationship. J neuroinflamm 2016; 13(1): 260.

6. Bahar E, Kim J-Y, Yoon H. Quercetin attenuates manganese-induced neuroinflammation by alleviating oxidative stress through regulation of apoptosis, iNOS/NF-KB and HO-1/Nrf2 pathways. Int J Mol Sci 2017; 18(9): 1989.

7. Lv H, Yu Z, Zheng Y, Wang L, Qin X, Cheng G, Ci X. Isovitexin exerts anti-inflammatory and anti-oxidant activities on lipopolysaccharide-induced acute lung injury by inhibiting MAPK and NF-KB and activating HO1/Nrf2 pathways. Int J Mol Sci 2016; 12(1): 72.

8. Minato K-i, Miyake Y, Fukumoto S, Yamamoto K, Kato Y, Shimomura $Y$, Osawa T. Lemon flavonoid, eriocitrin, suppresses exercise-induced oxidative damage in rat liver. Life Sci 2003; 72(14): 1609-1616.

9. Song $F$, Zhou L, Zhao J, Liu Q, Yang M, Tan R, Xu J, Zhang G, Quinn JM, Tickner J. Eriodictyol inhibits RANKL-induced osteoclast formation and function via inhibition of NFATc1 activity. J Cell Physiol 2016; 231(9): 1983-1993.
10. Lou $H$, Jing $X$, Ren $D$, Wei $X$, Zhang $X$. Eriodictyol protects against H2O2-induced neuron-like PC12 cell death through activation of Nrf2/ARE signaling pathway. Urochem Int 2012; 61(2): 251-257.

11. Naderali E, Nikbakht F, Ofogh SN, Rasoolijazi H. The role of rosemary extract in degeneration of hippocampal neurons induced by kainic acid in the rat: $A$ behavioral and histochemical approach. J Integr Neurosci 2018; 17(1): 31-43.

12. Health NIo: Guide for the care and use of laboratory animals: Natl Acad; 1985.

13. Noble LJ, Donovan F, Igarashi T, Goussev S, Werb Z. Matrix metalloproteinases limit functional recovery after spinal cord injury by modulation of early vascular events. J Neurosci 2002; 22(17): 7526-7535.

14. Choi DC, Lee JY, Lim EJ, Baik HH, Oh TH, Yune TY. Inhibition of ROS-induced p38MAPK and ERK activation in microglia by acupuncture relieves neuropathic pain after spinal cord injury in rats. Exp Neurol 2012; 236(2): 268-282.

15. Thuret S, Moon LD, Gage FH. Therapeutic interventions after spinal cord injury. Nat Rev Neurosci 2006; 7(8): 628.

16. PERIC M. The impact of early percutaneous tracheotomy on reduction of the incidence of ventilator associated pneumonia and the course and outcome of ICU patients. Signa Vitae2018; 14(1): 75-80.

17. Yang L, Jones NR, Blumbergs PC, Van Den Heuvel C, Moore EJ, Manavis J, Sarvestani GT, Ghabriel MN. Severity-dependent expression of pro-inflammatory cytokines in traumatic spinal cord injury in the rat. J Clin Neurosci 2005; 12(3): 276-284.

18. Zhang N, Wei G, Ye J, Yang L, Hong Y, Liu G, Zhong H, Cai $X$. Effect of curcumin on acute spinal cord injury in mice via inhibition of inflammation and TAK1 pathway. Pharmacol Rep 2017; 69(5): 1001-1006.

19. Liu J, Peng L, Li J. The Lipoxin A4 Receptor Agonist $B M L-111$ Alleviates Inflammatory Injury and Oxidative Stress in Spinal Cord Injury. Medical Science Monitor: Int Med J Expe Clin Res 2020; 26: e919883-919881.

20. Karova K, Wainwright JV, Machova-Urdzikova L, Pisal $R V$, Schmidt $M$, Jendelova $P$, Jhanwar-Uniyal $M$. Transplantation of neural precursors generated from spinal progenitor cells reduces inflammation in spinal cord injury via NF-KB pathway inhibition. J neuroinflamm 2019; 16(1): 12

21. Chen D, Pan D, Tang S, Tan Z, Zhang Y, Fu Y, Lü G, Huang Q. Administration of chlorogenic acid alleviates spinal cord injury via TLR4/NF-KB and p38 signaling pathway anti-inflammatory activity. Mol Med Rep 2018; 17(1): 1340-1346.

22. Zhang H, Liu Y-y, Jiang Q, Li K-r, Zhao Y-x, Cao C, Yao J. Salvianolic acid $A$ protects RPE cells against oxidative stress through activation of $\mathrm{Nrf} / \mathrm{HO}-1$ signaling. Free Radical Bio Med 2014; 69(219-228).

23. Sahin K, Tuzcu M, Gencoglu H, Dogukan A, Timurkan M, Sahin N, Aslan A, Kucuk O. Epigallocatechin-3-gallate activates Nrf2/HO-1 signaling pathway in cisplatin-

Trop J Pharm Res, August 2020; 19(8): 1616 
induced nephrotoxicity in rats. Life Sci 2010; 87(7-8): 240-245.

24. Shie P-H, Huang S-S, Deng J-S, Huang G-J. Spiranthes sinensis suppresses production of pro-inflammatory mediators by down-regulating the NF-KB signaling pathway and up-regulating $\mathrm{HO}-1 / \mathrm{Nrf2}$ anti-oxidant protein. Am J Chinese Med 2015; 43(05): 969-989.

25. Zhang J, Wang $Y$, Ji X, Shu Z. Tanshinone IIA protects against dopaminergic neuron degeneration via regulation of $\mathrm{DJ}-1$ and Nrf2/HO-1 pathways in a rodent model of Parkinson's disease. Trop J Pharm Res 2019; 18(5).

26. Lee SE, Yang $H$, Son GW, Park HR, Park C-S, Jin Y-H, Park YS. Eriodictyol protects endothelial cells against oxidative stress-induced cell death through modulating ERK/Nrf2/ARE-dependent heme oxygenase-1 expression. Int J Mol Sci 2015; 16(7): 14526-14539. 